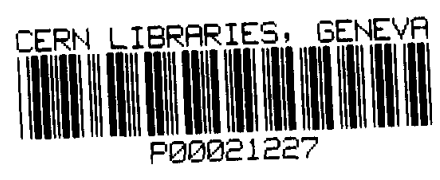

CERN-PPE/93-215

30.11 .1993

sw9406

\title{
PRECISION MEASUREMENT OF TWO IODINE LINES AT 585 NM AND 549 NM
}

\author{
R. Grieser, S. Dickopf, G. Huber, R. Klein, P. Merz \\ Institut für Physik, Universität Mainz \\ G. Bönsch, A. Nicolaus, H. Schnatz, \\ Physikalisch-Technische Bundesanstalt, Brauschweig
}

\begin{abstract}
The transition frequencies of the $\mathrm{i}$-component of the $\mathrm{R}(99) 15-1$ and the $\mathrm{w}$-component of the $R(85) 26-0$ transition in the B-X system of molecular ${ }^{127} \mathrm{I}_{2}$ have been determined with an overall relative standard uncertainty of $1,3 \cdot 10^{-10}$. For this purpose a commercial linear dye laser has been modified and stabilised to the corresponding iodine line. This dye laser serves as a transportable frequency standard which is compared with the wavelength standards of the PTB. The evaluation of an experiment for testing special relativity at the test storage ring (TSR) in Heidelberg is based on the precision of the reported interferometric wavelength comparison.
\end{abstract}


Precision measurement of two Iodine lines at $585 \mathrm{~nm}$ and $549 \mathrm{~nm}$

R.Grieser, S.Dickopf, G.Huber, R.Klein, P.Merz Institut für Physik, Universität Mainz G.Bönsch, A.Nicolaus, H.Schnatz

Physikalisch-Technische Bundesanstalt, Braunschweig 


\title{
Precision measurement of two Iodine lines at $585 \mathrm{~nm}$ and $549 \mathrm{~nm}$
}

\author{
R.Grieser ${ }^{1}$, S.Dickopf ${ }^{1}$, G.Huber ${ }^{1}$, R.Klein ${ }^{1}$, P.Merz ${ }^{1}$ \\ G.Bönsch ${ }^{2}$, A.Nicolaus ${ }^{2}$, H.Schnatz ${ }^{2}$ \\ 1 Institut für Physik, Universität Mainz, D-55099 Mainz, Fed.Rep.Germany \\ 2 Physikalisch-Technische Bundesanstalt (PTB),Bundesallee 100, D-338116 Braunschweig, \\ Fed.Rep.Germany
}

November 12, 1993

\begin{abstract}
The transition frequencies of the i-component of the $R(99) 15-1$ and the w-component of the $\mathrm{R}(85) 26-0$ transition in the B-X system of molecular ${ }^{127} I_{2}$ have been determined with an overall relative standard uncertainty of $1,3 \cdot 10^{-10}$. For this purpose a commercial linear dye laser has been modified and stabilized to the corresponding iodine line. This dye laser serves as a transportable frequency standard which is compared with the wavelength standards of the PTB. The evaluation of an experiment for testing special relativity at the test storage ring (TSR) in Heidelberg is based on the precision of the reported interferometric wavelength comparison.
\end{abstract}

\section{Introduction}

Laser spectroscopy at heavy ion storage rings gives rise to new precision experiments like a test of special theory of relativity, which is carried out at the Heidelberg test storage ring [1]. This experiment uses ${ }^{7} \mathrm{Li}^{+}$ions stored at high velocity $\left(\beta=\frac{v}{c}=0,064\right)$ as moving clocks with an optical resonance frequency $\nu_{0}$. For parallel and antiparallel excitation the corresponding Doppler shifted frequencies $\nu_{a}=\nu_{0} \gamma(1-\beta)$ and $\nu_{p}=\nu_{0} \gamma(1+\beta)$ are determined relative to ${ }^{127} I_{2}$ resonances. The accurate knowledge of $\nu_{0}, \nu_{a}, \nu_{p}$ allows a very sensitive test of the factor $\gamma=\frac{1}{\sqrt{1-\beta^{2}}}$. Any possible deviation of $\gamma$ will unbalance the relation $\nu_{a} \cdot \nu_{p}=\nu_{0}^{2}$ which holds strictly in special theory of relativity [2].

In this experiment helium like ${ }^{7} \mathrm{Li}^{+}$ions are excited in both ${ }^{3} S_{1}\left(F=\frac{3}{2}\right) \rightarrow{ }^{3} P_{2}\left(F=\frac{5}{2}\right)$ and ${ }^{3} S_{1}\left(F=\frac{5}{2}\right) \rightarrow{ }^{3} P_{2}\left(F=\frac{5}{2}\right)$ transitions, corresponding to $549 \mathrm{~nm}$ at rest [3]. For ions stored at $\frac{v}{c}=0,064$, the resonances are obtained with a parallel aligned $A r^{+}$-laser beam at $515 \mathrm{~nm}$ and a counterpropagating dye laser beam at $585 \mathrm{~nm}$. 
The $A r^{+}$laser is stabilized to the $\mathrm{P}(13) 43-0$ transition in ${ }^{127} I_{2}$ which is a recommended frequency standard [4], and its wavelength is known with very low uncertainty. ${ }^{127} I_{2}$ resonances are used to calibrate the dye laser's frequency at $585 \mathrm{~nm}$ and at $549 \mathrm{~nm}$ [5]. In order to determine these frequencies accurately, a transportable dye laser was modified for stabilization on ${ }^{127} I_{2}$. By means of the Michelson interferometer at the PTB in Braunschweig [6] the wavelength ratios relative to a Helium Neon laser stabilized to the i-component of the $R(127) 11-5$ transition in ${ }^{127} I_{2}$ at $633 \mathrm{~nm}$ have been measured.

\section{Experiment}

\subsection{The ${ }^{127} I_{2}$-stabilized dye laser}

A Coherent 599/21 linear dye laser was used, running on Rhodamine $B$ at $633 \mathrm{~nm}$, Rhodamine 6G at $585 \mathrm{~nm}$ and Rhodamine 110 at $549 \mathrm{~nm}$, pumped with a 1,4 W Ar${ }^{+}$-laser at $515 \mathrm{~nm}$. The short term stability was improved by the following modifications: a high pressure pump, a temperature stabilized cooler at $8^{\circ} \mathrm{C}$ and a sapphire jet nozzle were inserted into the dye circulation for a bubble free, low jitter operation. Moreover, the laser resonator was reinforced with two lateral invar rods reducing considerably the thermal drift and mechanical vibrations. With these improvements the laser was stabilized via the commercial servo loop to its reference cavity. A beat signal with a stabilized Helium Neon laser showed a FWHM of 1,6 MHz for integration times below $10 \mathrm{msec}$.

A separate servo loop was used for the long term stabilization of the reference cavity by means of saturated absorption. The laser was frequency modulated by adding the modulation voltage to the amplifier signal driving the piezo mounted mirror (tweeter). The voltage was adjusted to a modulation amplitude corresponding to $2,5 \mathrm{MHz}$.

In order to stabilize the laser frequency to a Doppler free absorption line, a part of the laser beam was sent through a single mode fiber to a saturation spectroscopy setup, mounted on a small iron base in an acoustically damed housing. A pump beam $\left(I=1 \frac{m W}{m m^{2}}, \emptyset_{1 / e}=0,8 m m\right)$ and a probe beam $\left(I=88 \frac{\mu W}{m^{2}}, \emptyset_{1 / e}=0,8 m m\right)$ were overlapped at an angle of $8 \mathrm{mrad}$ inside the iodine cell. The ${ }^{127} I_{2}$ cell was operated at a cold point temperature of $(6 \pm 0,3)^{\circ} \mathrm{C}$. To suppress the Doppler broadened absorption profile, the difference signal between the probe beam and the reference beam was observed, so that only the contribution of saturated absorption remained. This difference signal was filtered at the modulation frequency, synchronously detected with a lock-in amplifier, in- 


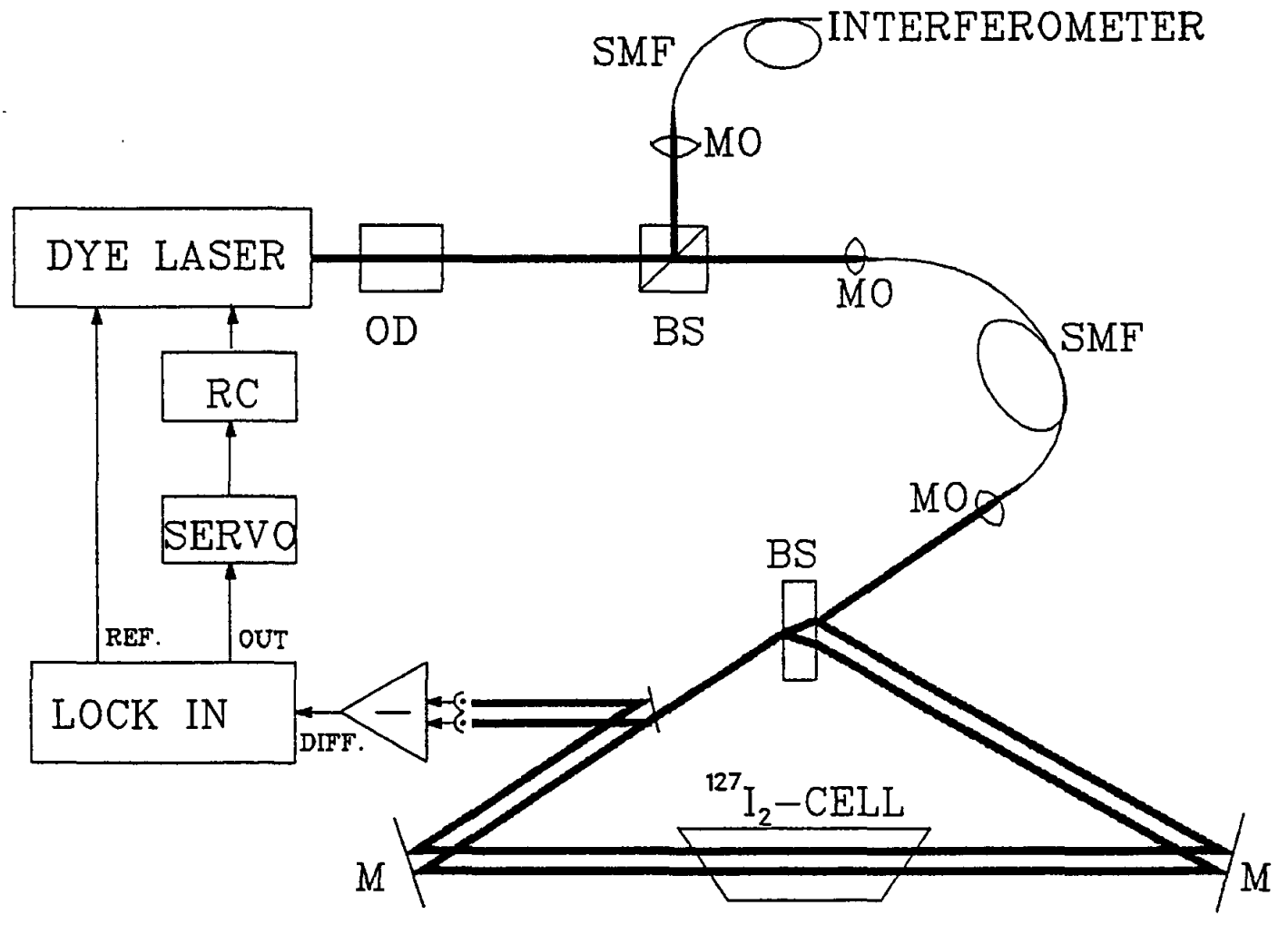

Figure 1: Set up for saturation spectroscopy with ${ }^{127} I_{2}$. A modified linear dye laser is used (COHERENT 599/21 with R6G or alternatively with R110). The dye laser beam is distributed after the optical diode $O D$ via a beamsplitter $B S$ and input couplers using microscope $(x 10)$ objectives MO and polarization preserving single mode fibers $S M F$, one to the interferometer and the other to a symmetrical saturation spectroscopy set up. The difference signal of the reference beam and the saturated beam is fed to a lock-in amplifier operating at $36 \mathrm{kHz}$ for $\mathrm{R} 110$ and $44 \mathrm{kHz}$ for R6G. The frequency modulation of the laser is obtained by dithering the tweeter mirror. The differential saturation signal is used to stabilize the reference cavity RC of the laser. 


\begin{tabular}{|l||c|}
\hline & $\begin{array}{c}\text { uncertainty contribution } \\
\mathrm{kHz}\end{array}$ \\
\hline Residual Doppler background & 43 \\
\hline Detuning of input filter & 30 \\
\hline Electronic offsets & 7,5 \\
\hline $\begin{array}{l}\text { Changes of the Iodine pressure } \\
\left.\text { (At } \mathrm{T}=6^{\circ} \mathrm{C}\right)\end{array}$ & $1^{\dagger}$ \\
\hline Power shifts & 5 \\
\hline Iodine cell impurities & $5^{\dagger}$ \\
\hline Recoil effect and second order Doppler shift & $1^{\dagger}$ \\
\hline Total & 54 \\
\hline
\end{tabular}

Table 1: Contributions to the total uncertainty of the Iodine stabilized dye laser, running at $549 \mathrm{~nm}$ or alternatively at $585 \mathrm{~nm}$. Estimations not given in the text and only concerning the external saturation spectroscopy are marked with ${ }^{\dagger}$ and taken from [7].

tegrated and used for long term stabilization of the reference cavity.

The stabilized laser system as shown in figure 1 has been compared with ${ }^{127} I_{2}$ stabilized He-Ne lasers at $633 \mathrm{~nm}$ at the Universität Mainz and at $543 \mathrm{~nm}$ at the PTB for the R6G and R110 operation with different mirror sets. The mirror sets showed different piezo resonances for the tweeter mirror, which made a change of the modulation frequency necessary ( $36 \mathrm{kHz}$ at $548 \mathrm{~nm}$ and $44 \mathrm{kHz}$ at $584 \mathrm{~nm}$ ). The $1 \mathrm{sec}$ average of the beat frequency showed a Gaussian distribution with $\sigma=1 \cdot 10^{-10}$.

The uncertainty contributions for the stabilized dye laser at both wavelengths are listed in table 1. The main contributions are a residual Doppler background, and a possible detuning of the frequency filter at the input channel of the lock-in amplifier, which leads to an asymmetric lineshape. This effect has been studied by comparison of the dye laser with the PTB lasers, and the estimated uncertainty component is $30 \mathrm{kHz}$. A residual Doppler background was estimated from the observed spectra to contribute by less than $43 \mathrm{kHz}$. Possible electronic offsets have been studied and drifts larger than $7,5 \mathrm{kHz}$ can be ruled out. An exchange of the Iodine absorption cell and power variations by a factor of 3 gave no detectable effect. The total uncertainty from table 1 corresponds to a relative standard uncertainty of the laser's frequency of $\frac{\Delta \nu}{\nu}<1 \cdot 10^{-10}$. 


\subsection{Reference lasers and Michelson interferometer}

For the wavelength determination a part of the dye laser's light was transmitted to the Michelson interferometer via a single mode fiber. Figure 2 shows in a simplified block diagram the whole experimental arrangement including all lasers necessary for the wavelength comparison which was carried out simultaneously with two reference lasers at 633 $\mathrm{nm}$ and $515 \mathrm{~nm}$. The light beams of the dye laser and two offset lasers at $633 \mathrm{~nm}$ and $515 \mathrm{~nm}$ were focussed into a single mode fiber for coupling the light to the interferometer. At the fiber end a parabolic mirror of $1 \mathrm{~m}$ focal length was used for collimating the beams. Only the central part of the expanded beam was transmitted through the interferometer. For the measurements, the path difference of the interferometer in both final positions of the movable mirror was locked to the interference minima of the dye laser's light, so that the mirror's displacement of about $3,8 \mathrm{~m}$ corresponded to an integer number of interference orders. The wavelengths of both offset lasers were locked to the stabilized path difference of the interferometer using interference minima of their own radiation. The fractional interference orders for the light of the ${ }^{127} I_{2}$-stabilized reference lasers were determined by beat frequency measurements between these lasers and the corresponding offset lasers. The integer interference orders were derived from the value of the mirror's displacement. This value was obtained from readings of a counting interferometer and an auxiliary measurement improving the accuracy, for which the Michelson interferometer was locked to a third ${ }^{127} I_{2}$-stabilized reference laser at $612 \mathrm{~nm}$. The displacement was evaluated from the measured fractional orders for the three known wavelengths. More details of the experimental conditions of the interferometric wavelength comparison are given in [6].

The ${ }^{127} I_{2}$-stabilized He-Ne reference laser at $633 \mathrm{~nm}$ was operated at the recommended conditions [11], partially with slightly increased uncertainties, i.e. $(15 \pm 0,3)^{\circ} \mathrm{C}$ coldpoint temperature of the iodine cell and $(6 \pm 0,5) \mathrm{MHz}$ peak to peak modulation, using an internal cell and the third harmonic locking technique. The laser was stabilized to the $i$ component of the $R(127) 11-5$ transition of ${ }^{127} I_{2}$ and its frequency was checked by comparison with another ${ }^{127} I_{2}$ - stabilized He-Ne laser before and after the measurements.

An $\mathrm{Ar}^{+}$laser stabilized by saturated absorption to the $a_{3}$ component of the $\mathrm{P}(13) 43-0$ transition of ${ }^{127} I_{2}$ was used as the second reference laser. It was operated with an external 


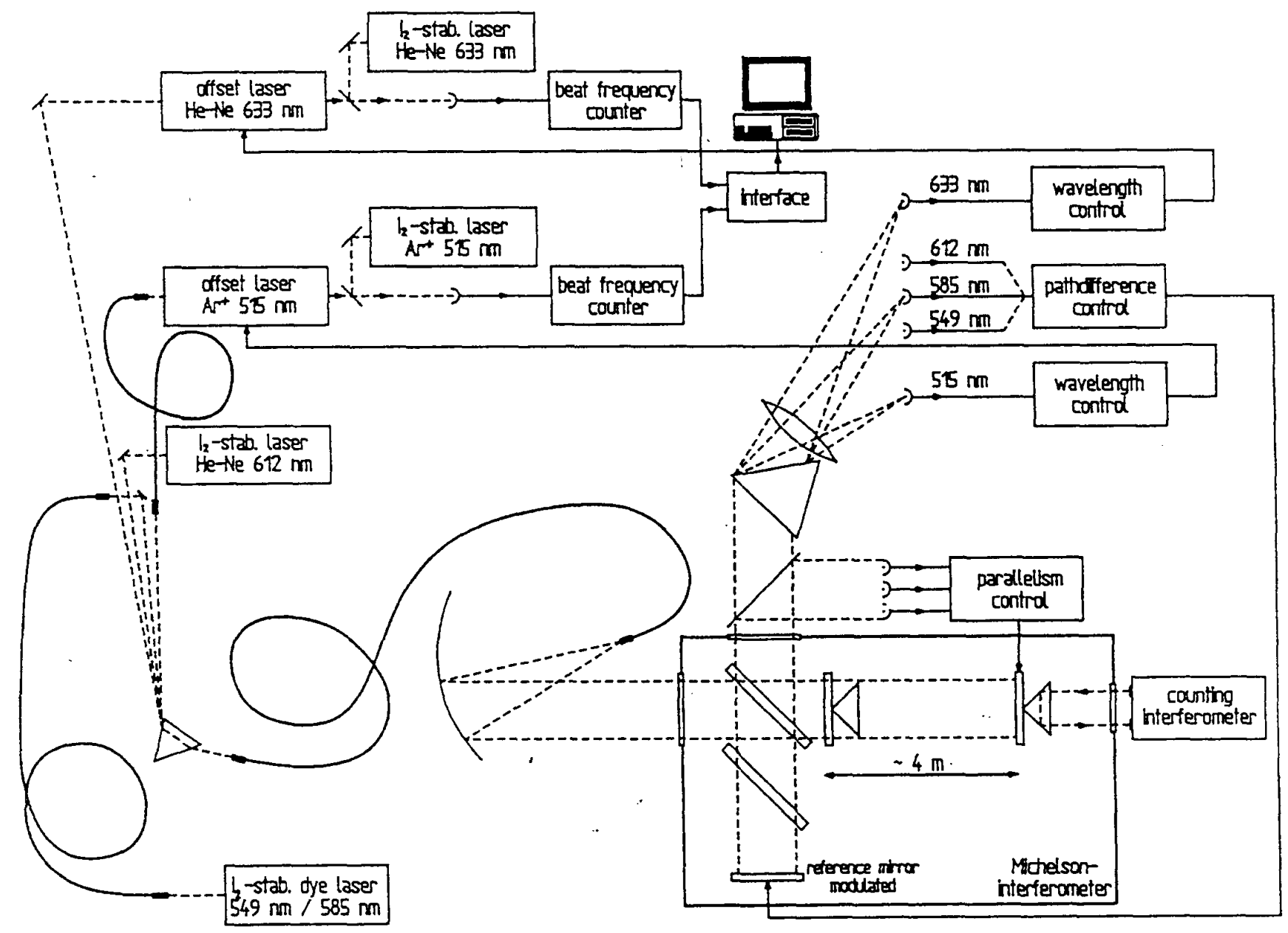

Figure 2: Scheme of the wavelength comparison. For details see text. 


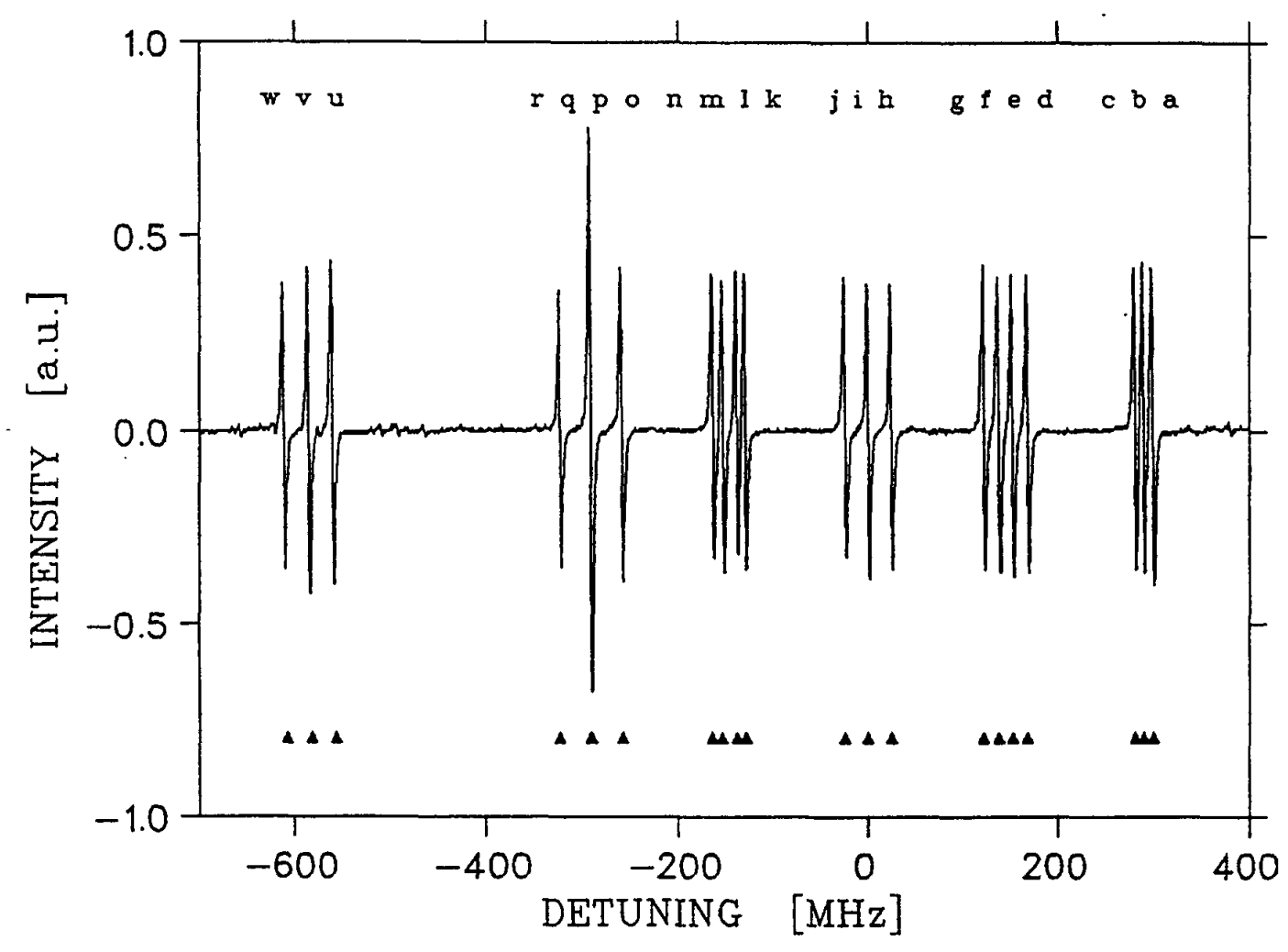

Figure 3: Saturation signals in ${ }^{127} I_{2}$ recorded at $584 \mathrm{~nm}$ by synchronous detection of the first harmonic difference signal with a frequency modulated laser as described in the text. The transition $B^{3} \Pi_{O u}^{+} \rightarrow X^{1} \Sigma_{g}^{+} R(99) 15-1$ is shown, and the $\mathrm{i}$-component is used as reference line. The triangles indicate the calculated positions of the hyperfine structure lines. The $q, p$ lines could not be resolved, because the spacing is below the modulation amplitude. The cold point temperature of the Iodine cell was kept at $(6 \pm 0,3)^{\circ} \mathrm{C}$.

iodine cell having a cold-point temperature of $(-5 \pm 0,3)^{\circ} \mathrm{C}$. The modulation width for the $3 f$-lock was adjusted to $(1,5 \pm 0,3) \mathrm{MHz}$ peak to peak. The power of the laser beam $(\emptyset=2 \mathrm{~mm})$ inside the iodine cell. was $1,8 \mathrm{~mW}$. The short term stability of the laser was improved by means of a reference cavity, the length of which was controlled by the iodine signals.

The estimated relative uncertainty originating from the interferometric part of the wavelength comparison amounts to $5 \cdot 10^{-11}$. The estimated relative standard uncertainty of the frequency of the He-Ne reference laser used for the experiment is $3 \cdot 10^{-11}$. The reproducibility of the $\mathrm{Ar}^{+}$reference laser under the conditions of the experiment is estimated to $2 \cdot 10^{-11}$. 


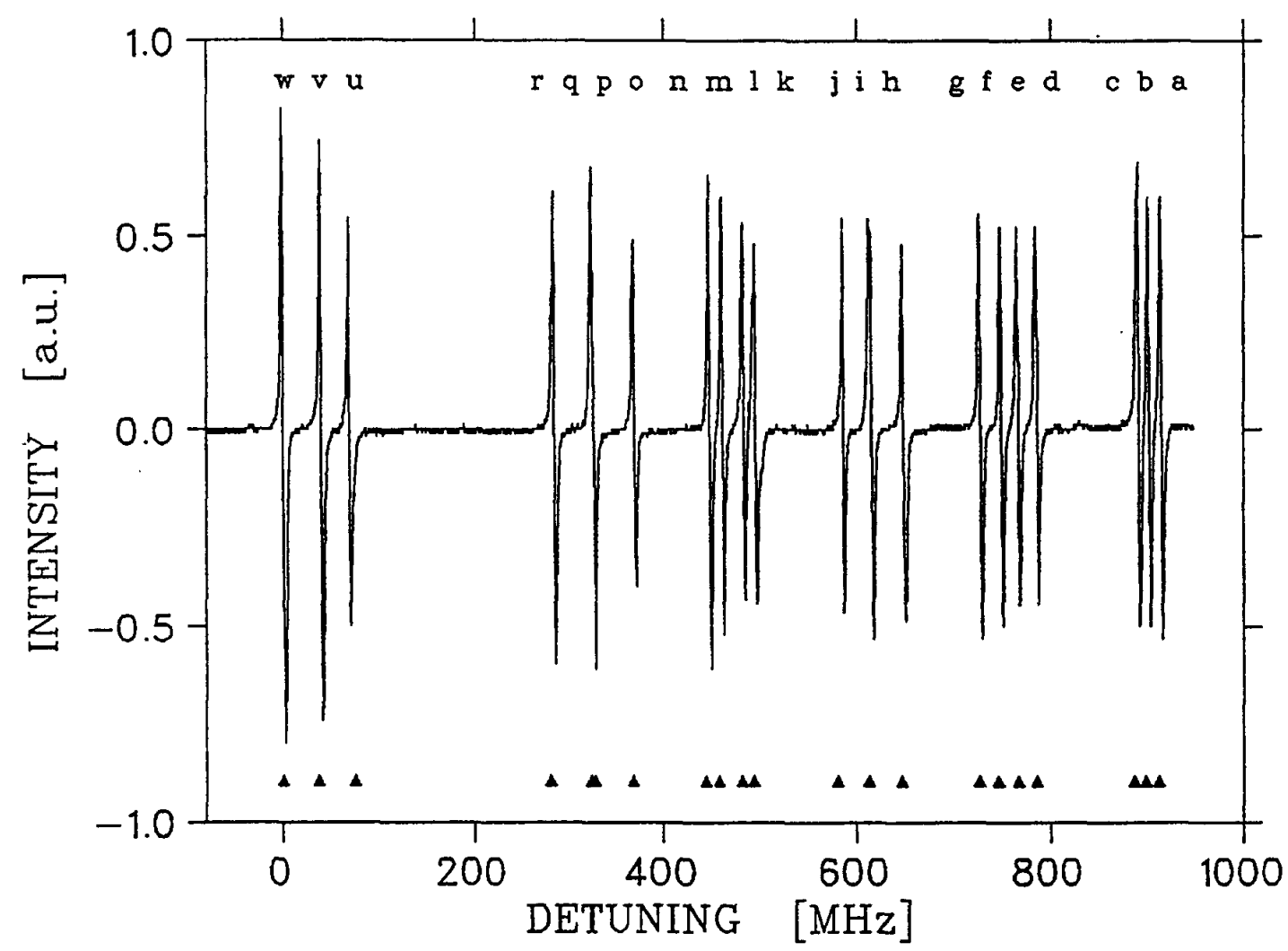

Figure 4: Saturation signals in ${ }^{127} I_{2}$ of the transition $B^{3} \Pi_{O u}^{+} \rightarrow X^{1} \Sigma_{g}^{+} R(85) 26-0$ at 549 $\mathrm{nm}$. The w-component is used as reference line. The hyperfine structure splitting of the $q, p$ lines is not resolved. The experimental procedure is identical to figure 3 .

\section{$3 \quad$ Results and discussion}

At $585 \mathrm{~nm}$ wavelength, the dye laser was stabilized to the $\mathrm{i}$-component of the $B^{3} \Pi_{O u}^{+} \rightarrow$ $X^{1} \Sigma_{g}^{+} R(99) 15-1$ transition, and at $549 \mathrm{~nm}$ to the w-component of the $R(85) 26-0$ transition in ${ }^{127} I_{2}$.

The coarse structure of the recorded spectra has been identified with the iodine atlas [8], whereas the hyperfine structure splitting has been calculated with a program of the Bureau International des Poids et Mesures [9] with the extrapolation formulas given by [10]. The calculated hyperfine structure splitting is shown in figures 3 and 4 by the position of the triangles.

The splitting has been measured by calibrating the free spectral range of a Fabry Perot interferometer, operated at $\mathrm{TEM}_{00}$ mode $(\mathrm{FSR}=606,8(1,9) \mathrm{MHz}$ ). This interferometer was stabilized to the $a_{3}$ component of ${ }^{127} I_{2}$ at $515 \mathrm{~nm}$, and the HFS splitting was compared with the separation of its transmission maxima. Due to non linearities in the scan 
operation of the laser the uncertainty of the measured splitting is below $2.6 \mathrm{MHz}$ within one free spectral range.

The interference orders measured with the Michelson Interferometer are used to calculate the wavelength ratios. Wavelength dependent corrections for the diffraction $\left(\leq 2 \cdot 10^{-11}\right)$ are included in the following values:

- $\frac{\lambda_{648}}{\lambda_{633}}=0,86668681714 \pm 13 \cdot 10^{-11}$

- $\frac{\lambda_{635}}{\lambda_{653}}=0,92381924206 \pm 13 \cdot 10^{-11}$

- $\frac{\lambda_{814}}{\lambda_{633}}=0,81308129593 \pm 7 \cdot 10^{-11}$

The total uncertainty is given by the contributions of the interferometric comparison and the lasers involved. With the recommended frequency value for the He-Ne reference laser $\nu_{633}=(473612214705 \pm 14) \mathrm{kHz}$ [11] the frequencies of the ${ }^{127} I_{2}$ - stabilized dye laser are calculated to

$$
\begin{aligned}
& \text { - } \nu_{549}=(546462926790 \pm 71) \mathrm{kHz} \\
& \text { - } \nu_{585}=(512667622777 \pm 67) \mathrm{kHz}
\end{aligned}
$$

All uncertainties given above correspond to one relative standard uncertainty $(1 \sigma)$.

The present results show a total relative uncertainty of $\sim 1,3 \cdot 10^{-10}$. With these measurements two new reference lines of iodine are available with uncertainties comparable to those of the recommended frequency standards [11]. Moreover, the wavelength ratio between the ${ }^{127} I_{2}$ stabilized Helium Neon laser at $633 \mathrm{~nm}$ and the ${ }^{127} I_{2}$ stabilized $\mathrm{Ar}^{+}$ laser has been redeterminated.

The uncertainty for the frequency standards in the test experiment of special theory of relativity is thus reduced by 3 orders of magnitude. In future experiments full advantage of this reduce uncertainty can be taken, provided that the ${ }^{7} \mathrm{Li}^{+}$transition frequencies at rest can be determined relative to the $R(85) 26-0{ }^{127} I_{2}$ line with the same accuracy.

In combination with the measurements in progress at the TSR the relative uncertainty reported in [1] will be decreased from $3 \cdot 10^{-5}$ to $3 \cdot 10^{-6}$.

The interferometric wavelength comparison has become the basis for frequency calibration in storage ring experiments, where the large Doppler shift of optical transitions are observed. The accurate knowledge of the ${ }^{127} I_{2}$ reference frequencies allows a precise determination of the ${ }^{7} \mathrm{Li}^{+}$velocity at the TSR for a laser and electron cooled beam to 
$\frac{\Delta v}{v}=10^{-7}$

Acknowledgements: The authors thank $H$. Darnedde for the loan of an $I_{2}$ stabilized Helium Neon laser and for checking its frequency against his standard. We gratefully acknowledge fruitful discussions with $F$. Riehle during the experiment.

\section{References}

[1] R.Klein et al., Z Phys. A 342, 455 (1992)

S.Schröder et al., Phys.Rev.Lett. 64, 2901 (1990)

[2] C.M.Will, PRD 45, 403 (1992)

M.Kretzschmar, Z Phys. A 342, 463 (1992)

[3] E.Riis et al., PRA 33, 3023 (1986)

[4] Documents concerning the New Definition of the Metre, Metrologia 19, 163, (1984)

[5] H.Rong et al., Z Phys. D 25, 337 (1993)

[6] G.Bönsch, Appl.Opt. 22(21), 3414 (1983)

G.Bönsch, IEEE Trans. Instrum. Meas. 34, 248 (1985)

G.Bönsch et al., PTB Mitteilungen, 99, 5-89 (1990)

[7] F.Spieweck, IEEE Trans.Instrum.Meas. 4,361 (1980)

[8] S.Gerstenkorn, P.Luc, Atlas du spectre d'absorption de la molecule d'iode 14800$20000 \mathrm{~cm}^{-1}$, Laboratoire Aime-Cotton, CNRS II, 1978

S.Gerstenkorn, P.Luc, Rev. Phys. Appl. 14, 791, (1979)

S.Gerstenkorn, P.Luc, Atlas du spectre d'absorption de la molecule d'iode 14800$20000 \mathrm{~cm}^{-1}$, Complement: Identification des transitions du systeme (B-X), Laboratoire Aime-Cotton, CNRS II, 1985

[9] S. Fredin-Picard, How to calculate hyperfine transitions energies, Technical Report BIPM-90/5, Bureau International des Poids et Mesures (1990)

[10] M.Gläser, Optics Communication 54, 335 (1985)

M.Gläser, Hyperfine Components of Iodine for optical frequency standards, PTBOpt-25, Braunschweig (1987) 
[11] Comité International des Poids et Mesures (CIPM), $81^{e}$ session, 1992, Recommendation 3 
